\title{
Three-dimensional analysis of bending losses in dielectric optical waveguides with arbitrary refractive-index profile
}

\author{
M. L. Calvo* \\ Visual Studies Center, School of Optometry, University of California at Berkeley, Berkeley, California 94720 \\ R. F. Alvarez-Estrada ${ }^{\dagger}$ \\ Theoretical Physics Group, Lawrence Berkeley Laboratory, University of California at Berkeley, Berkeley, \\ California 94720
}

Received May 15, 1986; accepted October 21, 1986

\begin{abstract}
A three-dimensional analysis of bending losses in dielectric optical waveguides is presented. It constitutes a nontrivial generalization of previous two- and three-dimensional studies by other authors. Our analysis is based on homogeneous integral equations for the total radiation field and suitable asymptotic approximations for Green's homogeneous integral equations for the total radiation field and suitable asy for a relevant Bessel function with large order and argument (the former being larger than the latter). A nontrivial check of the consistency of all those approximations is given. General formulas are presented for the radiated field and the energy flow and for a bending-loss coefficient in three dimensions. Numerical results are also given, in order to assess the difference between the results of other authors and ours. Such a difference is rather small for monomode behavior near cutoff, between the results of other authors and ours. Such a difference is rather small for monomose as as the parameter $V$ increases for a given core radius and propagation mode.
\end{abstract}

\section{INTRODUCTION}

Bending losses stand as an important phenomenon, giving rise to a decrease in the confined energy flow in an optical waveguide or fiber. A rather large number of interesting results $^{1-18}$ have been published on the subject. We refer (and will do so throughout the present work) to books by Marcuse ${ }^{19}$ and Snyder and Love ${ }^{20}$ as representative accounts of the basic theoretical understanding of curvature losses in optical waveguides, even if other works among those referred to above offer additional useful viewpoints. A comparative study of them ${ }^{19,20}$ reveals that each one displays basic insights, but it also suggests that further analysis is necessary. In fact, Marcuse's analysis ${ }^{19}$ contains some useful hints regarding the basic approximations in two dimensions, but even with certain three-dimensional extensions $^{6-11}$ it is restricted to special (even if important) cases, and, moreover, these works do not make use of the powerful and general framework offered by three-dimensional integral equations.

On the other hand, Snyder and Love ${ }^{20}$ do make use of three-dimensional integral equations as starting points and obtain some interesting approximate formulas, which, however, cannot be regarded as fully general three-dimensional versions of Marcuse's special two- and three-dimensional results referred to above. In this paper we will present a three-dimensional integral equation analysis in which suitable generalizations of Marcuse's approximations can indeed be carried out so as to obtain new and general formulas for the flow of radiated energy and bending-loss coefficients. In so doing, we shall generalize to three-dimensional optical fibers our previous work on bending losses in two-dimensional neutron waveguides. ${ }^{21}$
This paper is organized as follows. In Sections 2 and 3 we shall outline the basic equations and assumptions and some helpful properties for propagation modes in straight (unbent) fibers. In Section 4 we present the above-mentioned general treatment for the total radiation field in three-dimensional bent waveguides, based on a homogeneous integral equation and a suitable Green's function. A new and useful approximation for certain relevant Bessel functions of larger order and argument (the former being larger than the latter), which generalizes (but differs from) those used by other authors, is obtained: its derivation is given in Appen$\operatorname{dix}$ A. From this derivation a rather general representation is obtained for the total radiation field in the asymptotic region. In Section 5 we evaluate the flow of radiated energy for a general (multimode) curved waveguide and present a new formula for the bending-loss coefficient associated with any given propagation mode. In Section 6 , several numerical calculations are carried out in order to assess the magnitude of the main (exponential) factors determining the bending-loss coefficient. Throughout Sections 4, 6, and (in particular) 7 we discuss our results in comparison with those of previous authors, as summarized by Marcuse ${ }^{19}$ and Snyder and Love. ${ }^{20}$

\section{PHYSICAL FOUNDATIONS}

We consider in three-dimensional space a dielectric waveguide with infinite length. For the purpose of our study it has an $\bar{x}$-varying structure represented by the relative dielectric permittivity $\epsilon=\epsilon(\bar{x})[\bar{x}=(x, y, z)]$. (Throughout this work, vectors for three- and two-dimensional cases. will be designated by overbars and boldface, respectively.) The waveguide is surrounded by an infinite isotropic medium 
(the clad) with a constant relative permittivity $\epsilon_{c}$. Let an electromagnetic wave propagate along the waveguide; for simplicity, we shall consider a monochromatic optical field described by the electric and magnetic fields $\bar{E}(\bar{x}), \bar{H}(\bar{x})$ and the frequency $w$. We assume that $\epsilon(\bar{x})$ has a suitably smooth variation for $\bar{x}$ inside the waveguide. Thus, in a first study, Maxwell's equations can be approximated for any $\bar{x}$ as (timedependent factors will always be factored out and $k=w / c)$ :

$$
\begin{aligned}
& \left(\Delta+k^{2} \epsilon_{c}\right) \bar{E}=-k^{2}\left(\epsilon-\epsilon_{c}\right) \bar{E}, \\
& \left(\Delta+k^{2} \epsilon_{c}\right) \bar{H}=-k^{2}\left(\epsilon-\epsilon_{c}\right) \bar{H},
\end{aligned}
$$

with $\Delta=\Delta_{T}+\partial^{2} / \partial z^{2}, \Delta_{T}=\partial^{2} / \partial x^{2}+\partial^{2} / \partial y^{2}$, where $\epsilon-\epsilon_{c}>0$ $(=0)$ inside (outside) the waveguide. No use will be made (except in Section 5) of $\bar{\nabla}(\epsilon \bar{E})=0$ and $\bar{\nabla} \bar{H}=0$.

We also suppose that at the surface of the waveguide $\epsilon$ varies smoothly so that $\bar{E}, \bar{H}$, and the normal derivatives of their components are approximately continuous, in order to simplify our first analysis of bending losses.

For definiteness, $\epsilon$ and $\epsilon_{c}$ are real parameters (no absorption), but it will become obvious that our analysis will also be valid (up to at most some trivial modifications) for complex $\epsilon, \epsilon_{c}$.

To fix the ideas, we shall work only with the electric field $\bar{E}$ and its associated wave equation in Eq. (1a) in Sections 3-5 (and in Appendix B). A similar study will hold, obviously, for the magnetic field. Use of both $\bar{E}$ and $\bar{H}$ fields will be required in Section 5 for the analysis of energy flux and the bending-loss coefficient.

\section{UNBENT WAVEGUIDE (VANISHING CURVATURE)}

In a first step, we shall suppose that the waveguide is strictly straight (that is, it exhibits vanishing curvature), its axis being parallel to the $z$ axis and extending from $z=-\infty$ up to $z$ $=+\infty$.

Let $T$ be its transverse cross section in the $x y$ plane, in general with arbitrary geometry (this one will be supposed to be circular in the applications of Section 6; see Fig. 1). For convenience the $z$ axis and the origin $[\bar{x}=(0,0,0)]$ are chosen to lie outside the waveguide. Also, let $\epsilon(\bar{x})=\epsilon(x, y)$.

Under strict confinement conditions, a discrete number of propagation modes along the waveguide exist and are described by

$$
\bar{E}_{\mathrm{pm}}(\bar{x})=\exp (i \beta z) \bar{E}_{0}(\mathbf{x}), \quad \gamma^{2}=\beta^{2}-k^{2} \epsilon_{c} .
$$

For simplicity we shall devote most of our analysis to the case in which only one mode is propagating. The generalization to any superposition of propagation modes can be carried out without difficulty (see Section 5 for the most relevant details). The subscript pm denotes propagation mode. Also, $\beta$ is the real propagation constant $(\beta>0)$, and $\gamma>0$ is the modal parameter.

$\bar{E}_{0}(\mathbf{x})$ fulfills the homogeneous integral equation

$$
\bar{E}_{0}(\mathbf{x})=-(1 / 4 i) \int_{T} \mathrm{~d} \mathbf{x}^{\prime} H_{0}{ }^{(1)}\left(i \gamma\left|\mathbf{x}-\mathbf{x}^{\prime}\right|\right) k^{2}\left[\epsilon\left(\mathbf{x}^{\prime}\right)-\epsilon_{c}\right] \bar{E}_{0}\left(\mathbf{x}^{\prime}\right) .
$$

$H_{0}{ }^{(1)}$ is a Hankel function of zero order and first kind, here represented as [see Eq. (7.2.42) in Ref. 22]

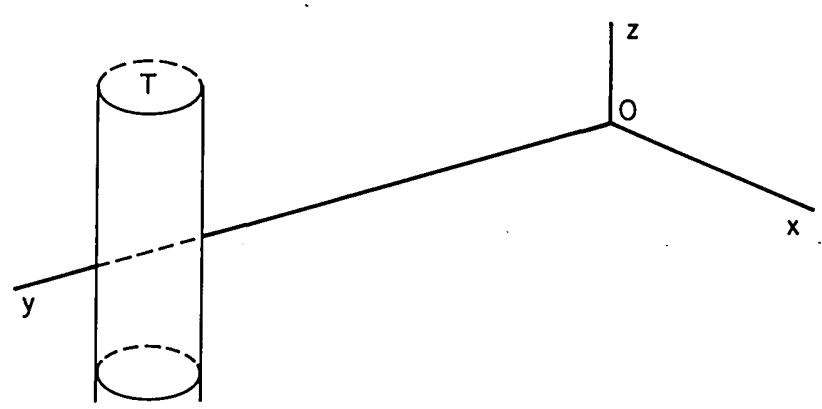

Fig. 1. Unbent three-dimensional waveguide with transverse cross section $T$. In this and the other figures $O$ denotes the origin of coordinates, far outside the fiber.

$$
\begin{aligned}
H_{0}{ }^{(1)}(i \gamma \mid \mathbf{x} & \left.-\mathbf{x}^{\prime} \mid\right) \\
& =-4 i \int \frac{\mathrm{d} l_{x} \mathrm{~d} l_{y}}{(2 \pi)^{2}} \frac{\exp \left\{i\left[l_{x}\left(x-x^{\prime}\right)+l_{y}\left(y-y^{\prime}\right)\right]\right\}}{l_{x}^{2}+l_{y}{ }^{2}+\gamma^{2}},
\end{aligned}
$$

with

$$
\left(-\Delta_{T}+\gamma^{2}\right) \frac{i}{4} H_{0}^{(1)}\left(i \gamma\left|\mathbf{x}-\mathbf{x}^{\prime}\right|\right)=\delta^{(2)}\left(\mathbf{x}-\mathbf{x}^{\prime}\right),
$$

$\delta^{(2)}\left(\bar{x}-\bar{x}^{\prime}\right)$ being a two-dimensional delta function. $H_{0}{ }^{(1)}\left(i \gamma\left|\mathbf{x}-\mathbf{x}^{\prime}\right|\right)$ is, in fact, a two-dimensional Green's function. Equations (3) and (4) will both be useful later, particularly for checking the correctness of our solution in the bent-fiber analysis.

\section{BENT WAVEGUIDE: RADIATION FIELD}

\section{A. Homogeneous Integral Equation for the Total Field}

We shall now consider that the three-dimensional waveguide is perfectly straight from $z=-\infty$ up to a finite distance $z_{0}\left(z_{0}<0\right.$ and is large in magnitude), that it lies far from the origin, and that it has a large curvature radius $R$, for $z_{0}<z<$ $+\infty$. The dominant curvature effect occurs mainly in the $x z$ plane far from the origin (see Fig. 2).

A bound field propagating along the $-\infty<z<z_{0}$ section of the waveguide fulfills, in principle, the confinement conditions established in Eqs. (2) and (3) even if, for $z>z_{0}$, where the waveguide is bent, a certain percentage of energy is lost as it escapes from the bending region towards the clad. Thus the natural way to formulate the field, because of the actual incoming wave conditions and the fact that $\epsilon-\epsilon_{c}$ never vanishes along the infinite waveguide, leads to a homogeneous integral equation.

Specifically, the total field $\bar{E}$ can be expressed as the homogeneous integral equation

$$
\bar{E}(\bar{x})=\int \mathrm{d}^{3} \overline{x^{\prime}} G\left(\bar{x}-\bar{x}^{\prime}\right) k^{2}\left[\epsilon\left(\overline{x^{\prime}}\right)-\epsilon_{\mathrm{c}}\right] \bar{E}\left(\bar{x}^{\prime}\right),
$$

where $G$ is the three-dimensional Green's function [ $(-\Delta-$ $\left.k^{2} \epsilon_{c}\right) G\left(\bar{x}-x^{\prime}\right)=\delta^{(3)}\left(\bar{x}-x^{\prime}\right) ; \eta \rightarrow 0^{+}, \bar{l}=\left(l_{\mathrm{x}}, l_{\mathrm{y}}, l_{\mathrm{z}}\right),-\infty<l_{h}<$ $+\infty, h=x, y, z]$

$$
\begin{aligned}
G\left(\bar{x}-\bar{x}^{\prime}\right) & =(1 / 2 \pi)^{3} \int \mathrm{d}^{3} l \frac{\exp \left[i \bar{l}\left(\bar{x}-\bar{x}^{\prime}\right)\right]}{l^{2}-\left(k^{2} \epsilon_{c}+i \eta\right)} \\
& =(1 / 4 \pi) \frac{\exp \left(i k \epsilon_{c}{ }^{1 / 2}\left|\bar{x}-\bar{x}^{\prime}\right|\right)}{\left|\bar{x}-\bar{x}^{\prime}\right|} .
\end{aligned}
$$


A check of consistency of Eqs. (5) and (6) follows directly. For $R \rightarrow \infty$ (unbent waveguide), by using $\epsilon\left(\overline{\mathbf{x}}^{\prime}\right) \rightarrow \epsilon\left(\mathbf{x}^{\prime}\right)$, substituting Eqs. (2)-(6) into Eq. (5), and integrating over $z$ and $l_{y}$ conveniently, the representation given in Eq. (3) is found. Thus the unbending case can be obtained as a particular limit of the bending case.

\section{B. Asymptotic Expansion}

Owing to the particular geometry of the problem, the possible values of $y^{\prime}$, as $\bar{x}^{\prime}$ varies inside the waveguide, are small (see Fig. 2). For given $\bar{x}^{\prime}$ we fix the direction of $\bar{x}$ close enough to the $x z$ plane, thus forming an angle $\theta$ with the $y$

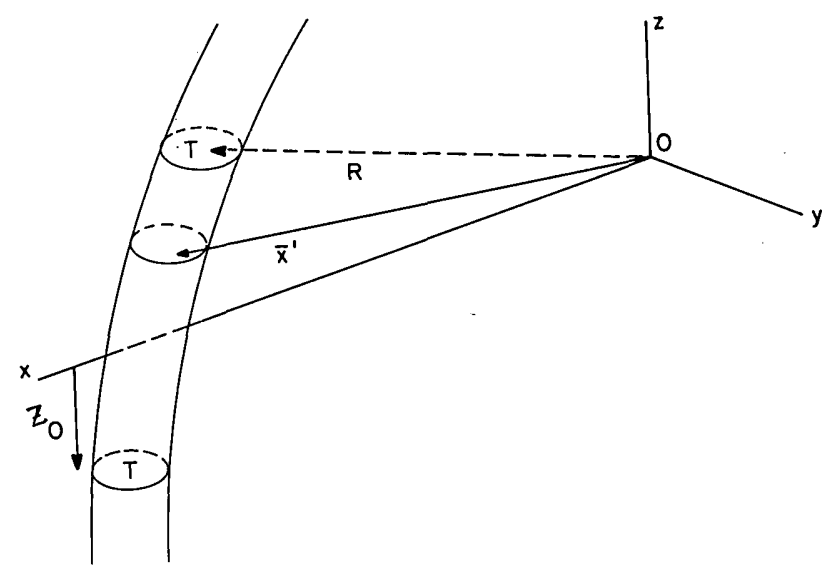

Fig. 2. Bent three-dimensional fiber. The part of the waveguide in $-\infty<z<z_{0}$ (lower part) is perfectly straight. The dashed line represents one curvature radius $R$. The part above $Z_{0}$ (upper part) is curved. Since $R$ is large, the transverse cross sections above and below $Z_{0}$ are approximately equal. Since the origin lies far from the waveguide, the range of possible values of $y^{\prime}$ as $x^{\prime}$ varies inside it is quite small, which justifies the approximations made in expression (8). axis close to $\pi / 2$ (see Fig. 3). Provided that $|\bar{x}| \gg R$, it is then permissible to replace the Green's function $G$ by its asymptotic approximation

$$
G\left(\bar{x}-\bar{x}^{\prime}\right) \simeq(1 / 4 \pi|\bar{x}|) \exp \left(i k \epsilon_{c}{ }^{1 / 2}|\bar{x}|\right) \exp \left(-i \bar{k}^{\prime} \bar{x}^{\prime}\right),
$$

with

$$
\bar{k}^{\prime} / k \epsilon_{\mathrm{c}}{ }^{1 / 2}=\bar{x} /|\bar{x}|=(\cos \varphi \sin \theta, \cos \theta, \sin \varphi \sin \theta) .
$$

Here and in what follows, $\varphi$ and $\varphi^{\prime}$ are the angles formed by the two-dimensional vectors $(x, z)$ and $\left(x^{\prime}, z^{\prime}\right)$, respectively, with the $x$ axis:

$$
\begin{aligned}
& |z|_{x}=\left(x^{2}+z^{2}\right)^{1 / 2} \sin \varphi \\
& \left|z^{\prime}\right|_{x}=\left(x^{\prime 2}+z^{\prime 2}\right)^{1 / 2} \sin \varphi^{\prime} .
\end{aligned}
$$

Since $\theta \simeq \pi / 2$ and the possible values of $y^{\prime}$ are small, one has

$$
\begin{aligned}
\bar{k}^{\prime} \bar{x}^{\prime} & \simeq u \cos \left(\varphi-\varphi^{\prime}\right), \\
\left|\bar{x}^{\prime}\right| & \simeq\left(x^{\prime 2}+{z^{\prime}}^{2}\right)^{1 / 2} ; u=k \epsilon_{c}{ }^{1 / 2} \sin \theta\left(x^{\prime 2}+{z^{\prime}}^{2}\right)^{1 / 2} .
\end{aligned}
$$

It is possible to use the standard two-dimensional partialwave equation [see, for instance, Eqs. (9.1.44) and (9.1.45) in Ref. 23]

$$
\exp \left(-i \bar{k}^{\prime} \bar{x}^{\prime}\right) \simeq \sum_{M=-\infty}^{+\infty} \exp [i(\pi / 2) M] \exp \left[i M\left(\varphi-\varphi^{\prime}\right)\right] J_{|M|}(u),
$$

where $J_{|M|}$ is a Bessel function of order $|M|$.

The wave front $\exp \left(-i \bar{k}^{\prime} \bar{x}^{\prime}\right)$ is now interpreted as a discrete infinite superposition of phase terms modulated by oscillatory cylindrical functions. The degree of oscillation depends on the $u$ argument.

For large $R$ and consistent with the above approximation,

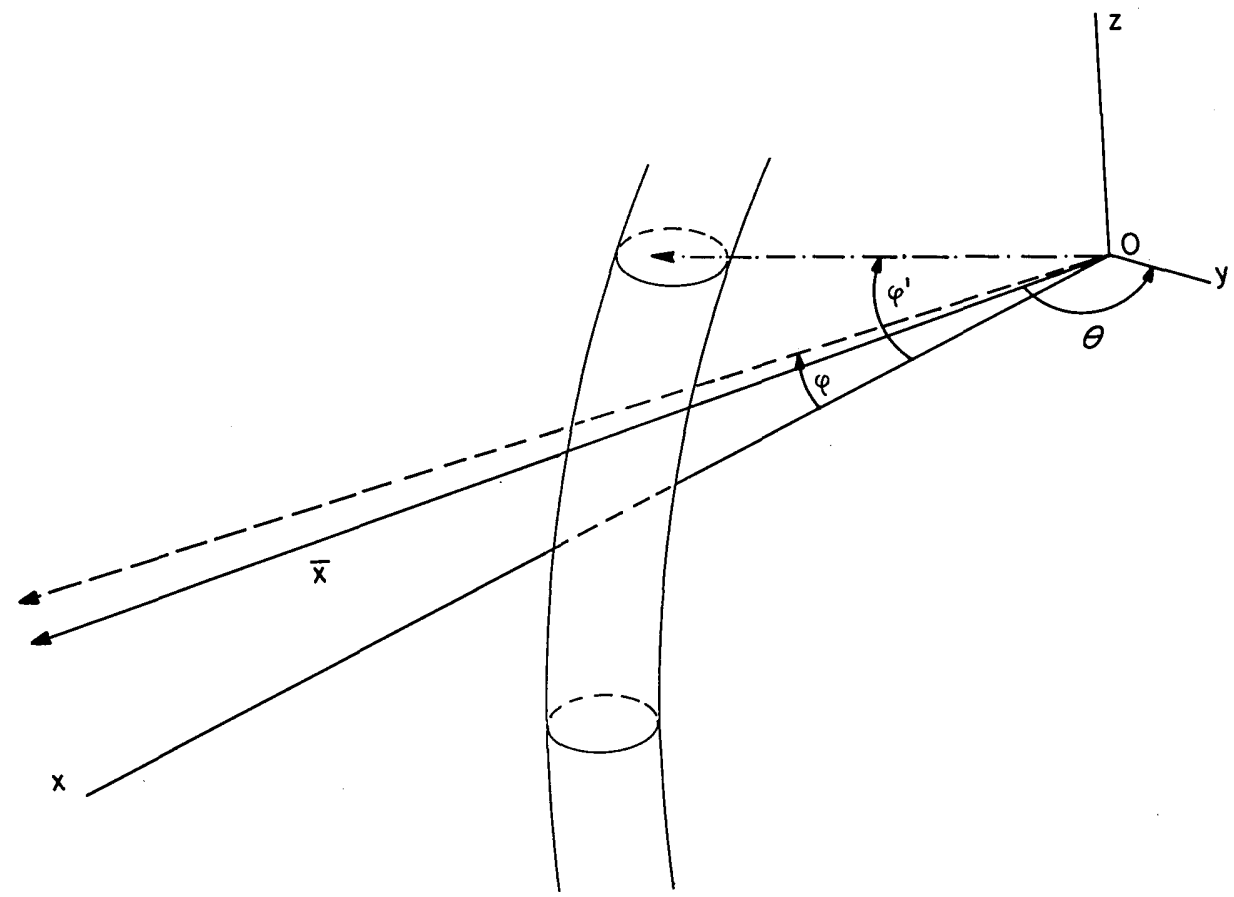

Fig. 3. The angle formed by $\bar{x}(|\bar{x}| \gg R$ ) with the $y$ axis is $\theta$ (close to $\pi / 2$ ). The projection of $\bar{x}$ on the $x z$ plane is indicated by a dashed vector, and $\varphi$ is the angle between the $x z$ plane and the $x$ axis. The vector represented by $(-\cdot-\cdots)$ is the projection of $\bar{x}^{\prime}$ (not displayed) on the $x z$ plane: the angle between such a projection and the $x$ axis is $\varphi^{\prime}$. 


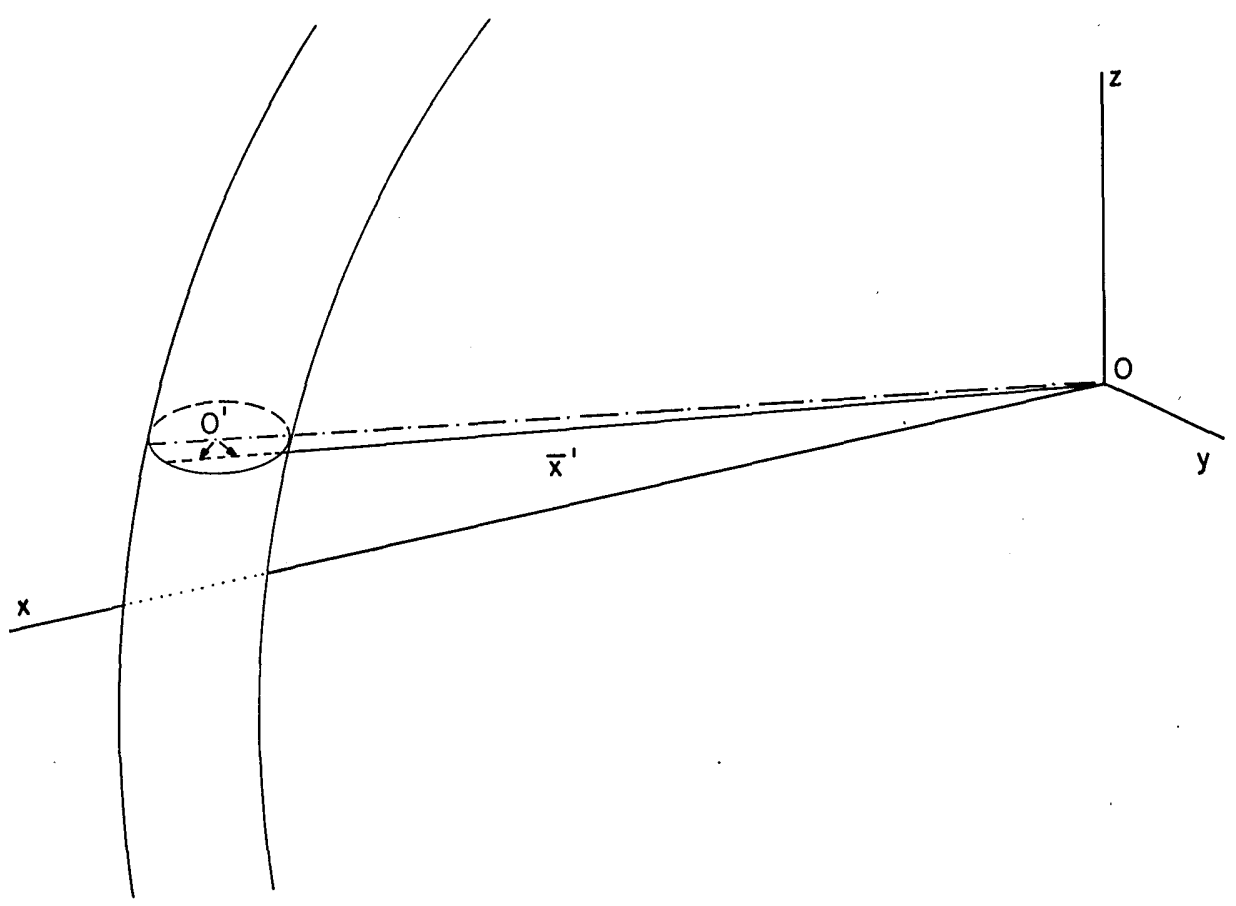

Fig. 4. New local coordinates are defined with respect to some new origin $O^{\prime}$ lying inside the transverse cross section $T$. The distance between the new $\left(\mathrm{O}^{\prime}\right)$ and the former $(O)$ origins is $R$. A short vector would have local coordinates $\left(x_{1}^{\prime} y^{\prime}\right)$ with respect to the new origin $\mathrm{O}^{\prime}$.

the following additional approximations will be performed on the right-hand sides of Eqs. (5) and (9):

(1) Introduce a local vector $\left(x_{1}{ }^{\prime}, y^{\prime}\right)$ as the new coordinates of any point in the transverse cross section $T$ of the waveguide with respect to a new origin lying inside $T$ (see Fig. 4). As $R$ is assumed to be suitably large, $\left|x_{1}{ }^{\prime}\right|<R$ holds for any point inside $T$. It is then possible to approximate

$$
\left[x^{\prime 2}+z^{\prime 2}\right]^{1 / 2} \simeq R+x_{1}^{\prime} .
$$

(2) The confined modal field defined in Eq. (2) is now interpreted as a local modal field, and then

$$
\bar{E}\left(\bar{x}^{\prime}\right) \simeq \exp \left(i \beta z^{\prime}\right) \vec{E}_{0}\left(x_{1}{ }^{\prime}, y^{\prime}\right)=\bar{E}_{\mathrm{pm}} ;
$$

also, $\epsilon\left(\overline{x^{\prime}}\right) \simeq \epsilon\left(x_{1}^{\prime}, y^{\prime}\right)$.

In both approximations, $\beta, \bar{E}_{\mathrm{pm}}, \bar{E}_{0}\left(x_{1}{ }^{\prime}, y^{\prime}\right)$, and $\epsilon\left(x_{1}{ }^{\prime}, y^{\prime}\right)$ satisfy Eqs. (2)-(4) and so correspond to an exact propagation mode of the perfect straight waveguide.

(3) Assume $\varphi^{\prime}$ to be small, set $z^{\prime} \simeq R \varphi^{\prime}$ in all exponentials [but not in the $u$ argument of $J$ 's, which will require a better approximation based on approximation (1); see below], and let $\int_{-\infty}^{+\infty} \mathrm{d} z^{\prime} \rightarrow R \int_{0}^{2 \pi} \mathrm{d} \varphi^{\prime}$.

For fixed $|\vec{x}|(\gg R), \theta$ (close to $\pi / 2$ ), and $\varphi$, we replace expressions (7) and (9) into the right-hand side of Eq. (5), use the above approximations, integrate over $\varphi^{\prime}$, and realize that only $M \simeq \beta R$ contributes (that is, take $\beta R$ to be either an integer exactly or that integer $M$ which is closest to the large dimensionless number $\beta R$ ). Thus Eq. (5) yields the asymptotic representation

$$
\bar{E}(\bar{x}) \simeq(1 /|\bar{x}|) \exp \left[i\left(k \epsilon_{c}{ }^{1 / 2}|\bar{x}|+\beta R \varphi+\pi \beta R / 2\right)\right] R \bar{e},
$$

with

$$
\bar{e}=1 / 2 \int_{T} \mathrm{~d} x_{1}^{\prime} \mathrm{d} y^{\prime} J_{\beta R}(u) k^{2}\left[\epsilon\left(x_{1}^{\prime}, y^{\prime}\right)-\epsilon_{0}\right] \bar{E}_{0}\left(x_{1}^{\prime}, y^{\prime}\right),
$$

where no approximations have been carried out, so far, regarding $J_{\beta R}(u)$.

The above treatment, which led from Eq. (5) to expression (10) and Eq. (11), is essentially parallel to that described in Ref. 20, with some specific differences. The most important one is that they approximate $\left(x^{\prime 2}+z^{2}\right)^{1 / 2}$ by $R$ in the $u$ argument of $J_{\beta R}$. They then proceed directly to evaluate the power radiated by the bent waveguide (considered in that case as a bent antenna), and the resulting integral is asymptotically calculated as

$$
\begin{aligned}
J_{\beta R}\left(k \epsilon_{c}{ }^{1 / 2} \sin \theta R\right) \simeq & (1 / 2 \pi R)^{1 / 2}\left[1 /\left(\beta^{2}-k^{2} \epsilon_{c} \sin ^{2} \theta\right)^{1 / 4}\right] \\
& \times \exp \left\{-1 / 3\left(\beta R / \sin ^{3} \theta\right)\right. \\
& \left.\times\left[\left(\beta^{2} / k^{2} \epsilon_{c}\right)-\sin ^{2} \theta\right]^{3 / 2}\right\} .
\end{aligned}
$$

In the $J_{\beta R}$ function, $\beta R>k \epsilon_{c}{ }^{1 / 2} \sin \theta R$, as $R$ is always large (see below). We shall replace $J_{\beta R}(u)$ by an improved approximation, leading to related, but different, results. Our method essentially constitutes a nontrivial three-dimensional generalization of the procedure devised by Marcuse for two dimensions. ${ }^{15}$

Our basic new three-dimensional approximation is, for any $x_{1}{ }^{\prime}$ that is being integrated over in Eq. (11),

$$
J_{\beta R} \simeq \exp [\beta R \Lambda(\theta)] /\left[2 \pi R \gamma_{1}(\theta)\right]^{1 / 2} \exp \left[x_{1}{ }^{\prime} \gamma_{1}(\theta)\right],
$$

with

$$
\gamma_{1}(\theta)=\left(\beta^{2}-k^{2} \epsilon_{c} \sin ^{2} \theta\right)^{1 / 2}
$$

and

$$
\left.\left.\Lambda(\theta)=\beta^{-1} \gamma_{1}(\theta)-\tanh ^{-1}\right] \beta^{-1} \gamma_{1}(\theta)\right] .
$$

Notice that our results differ from Eq. (12). The proof of expression (13) is outlined in Appendix A. 
On combining Eq. (11) and expression (13), we get the new formula for the total radiation field to be used in the computation of the three-dimensional bending losses:

$$
\bar{e}=1 / 2 \frac{\exp [\beta R \Lambda(\theta)]}{\left[2 \pi R \gamma_{1}(\theta)\right]^{1 / 2}} \bar{e}_{1}
$$

with

$$
\bar{e}_{1}=\int_{T} \mathrm{~d} x_{1}{ }^{\prime} \mathrm{d} y^{\prime} \exp \left[x_{1}^{\prime} \gamma_{1}(\theta)\right] k^{2}\left[\epsilon\left(x_{1}^{\prime}, y^{\prime}\right)-\epsilon_{c}\right] \bar{E}_{0}\left(x_{1}{ }^{\prime}, y^{\prime}\right)
$$

\section{ENERGY FLOW IN A BENT WAVEGUIDE}

We shall study the incoming energy flow $F_{\text {in }}$ and the energy radiated by the bent fiber $F_{\text {rad }}$, and we shall derive a formula for the bending-loss coefficient.

We start by recalling the time-averaged Poynting vector ${ }_{1}^{1} / 2 \operatorname{Re}\left(\bar{E} \times \bar{H}^{*}\right)$. The incoming propagation mode is fully described, in $-\infty<z<z_{0}$, by the electric field [Eq. (2)] and the magnetic field $\bar{H}_{\mathrm{pm}}=\exp (i \beta \mathrm{z}) \bar{H}_{0}(\mathbf{x}, y)$ [which satisfies equations similar to Eqs. (2) and (3), with $\bar{E}_{\mathrm{pm}}$ and $\bar{E}_{0}$ replaced by $\bar{H}_{\mathrm{pm}}$ and $\bar{H}_{0}$, respectively]. The incoming energy flow along the $z$ axis across any plane perpendicular to the latter in $-\infty<z<z_{0}$ is $\left[\bar{u}_{z}=(0,0,1), \bar{E}_{0}=\left(E_{0 x}, E_{0 y}, E_{0 z}\right)\right.$, etc. $]$;

$$
\begin{aligned}
F_{\text {in }} & =\iint_{-\infty}^{+\infty} \mathrm{d} x_{1}{ }^{\prime} \mathrm{d} y^{\prime} \bar{u}_{z}{ }^{1 / 2} \operatorname{Re}\left(\vec{E}_{\mathrm{pm}} \times \bar{H}_{\mathrm{pm}}{ }^{*}\right) \\
& =\iint_{-\infty}^{+\infty} \mathrm{d} x_{1}{ }^{\prime} \mathrm{d} y^{\prime}{ }^{1} / 2 \operatorname{Re}\left(E_{0 x} H_{0 y}{ }^{*}-E_{0 y} H_{0 x}{ }^{*}\right) .
\end{aligned}
$$

In order to evaluate $F_{\text {rad }}$, we require, for $|\bar{x}| \gg R$ and $\theta$ close to $\pi / 2$, the asymptotic expression for the electric field given through expression (10) and Eqs. (15) and (16) together with a similar one for the magnetic field (which can be derived similarly), namely,

$$
\bar{H}(\bar{x}) \simeq(1 /|\bar{x}|) \exp \left\{i\left[k \epsilon_{c}{ }^{1 / 2}|\bar{x}|+\beta R \varphi+\pi / 2 \beta R\right]\right\} R \bar{h},
$$

with

$$
\bar{h}=1 / 2 \exp [\beta R \Lambda(\theta)]\left[2 \pi R \gamma_{1}(\theta)\right]^{1 / 2} \bar{h}_{1}
$$

and

$$
\bar{h}_{1}=\int_{T} \mathrm{~d} x_{1}^{\prime} \mathrm{d} y^{\prime} \exp \left[x_{1}^{\prime} \gamma_{1}(\theta)\right] k^{2}\left[\epsilon\left(x_{1}^{\prime}, y^{\prime}\right)-\epsilon_{0}\right] \bar{H}_{0}\left(x_{1}{ }^{\prime}, y^{\prime}\right)
$$

At this point we shall outline the essentials of the calculation of the radiated energy flow corresponding to a multimode behavior. Consider, for $z<z_{0}$, a linear superposition of propagation modes $\Sigma_{\alpha=0}^{N} c_{\alpha} \bar{E}_{\mathrm{pm}_{\alpha}}, \sum_{\alpha=0}^{N} c_{\alpha} \bar{H}_{\mathrm{pm}_{\alpha}}, c_{\alpha}$ being some given numerical coefficients and $\bar{E}_{\mathrm{pm}_{\alpha}}$ fulfilling Eq. (2) with certain $\beta_{\alpha}, \gamma_{\alpha}, \bar{E}_{0 \alpha}$, etc. The total fields are then $\vec{E}=$ $\Sigma_{\alpha=0}^{N} c_{\alpha} \bar{E}_{\alpha}, \bar{H}=\Sigma_{\alpha=0}^{N} c_{\alpha} \bar{H}_{\alpha}$ [where $\bar{E}_{\alpha}$ satisfies Eq. (5), etc.]. The asymptotic behaviors of $\bar{E}_{\alpha}$ and $\bar{H}_{\alpha}$ are given in expressions (10) and (18) and Eqs. (15), (16), (19), and (20) in terms of $\Xi_{\alpha}(\theta), \gamma_{1 \alpha}(\theta), E_{0 \alpha}$, and $\bar{H}_{0 \alpha}$.

The radiated energy flow through a finite solid angle $\Omega$ on the spherical surface of large radius $|\bar{x}|(|\bar{x}| \gg R)$, the center of which is at $\bar{x}=(0,0,0)$, and determined by the angles $\pi / 2$ $-\theta_{0}<\theta<\pi / 2+\theta_{0}$ and $\varphi_{1}<\varphi<\varphi_{2}$, where $\theta_{0}$ is small and $\varphi_{1}$ and $\varphi_{1}$ will be discussed later, is $(\mathrm{d} \Omega=\mathrm{d} \varphi \sin \theta \mathrm{d} \theta$; see Fig. 5)

$$
F_{\mathrm{rad}}=|\bar{x}|^{2} \int_{\Omega} \mathrm{d} \Omega(\bar{x} /|\bar{x}|)^{1} /{ }_{2} \operatorname{Re}\left(\bar{E} \times \bar{H}^{*}\right) .
$$

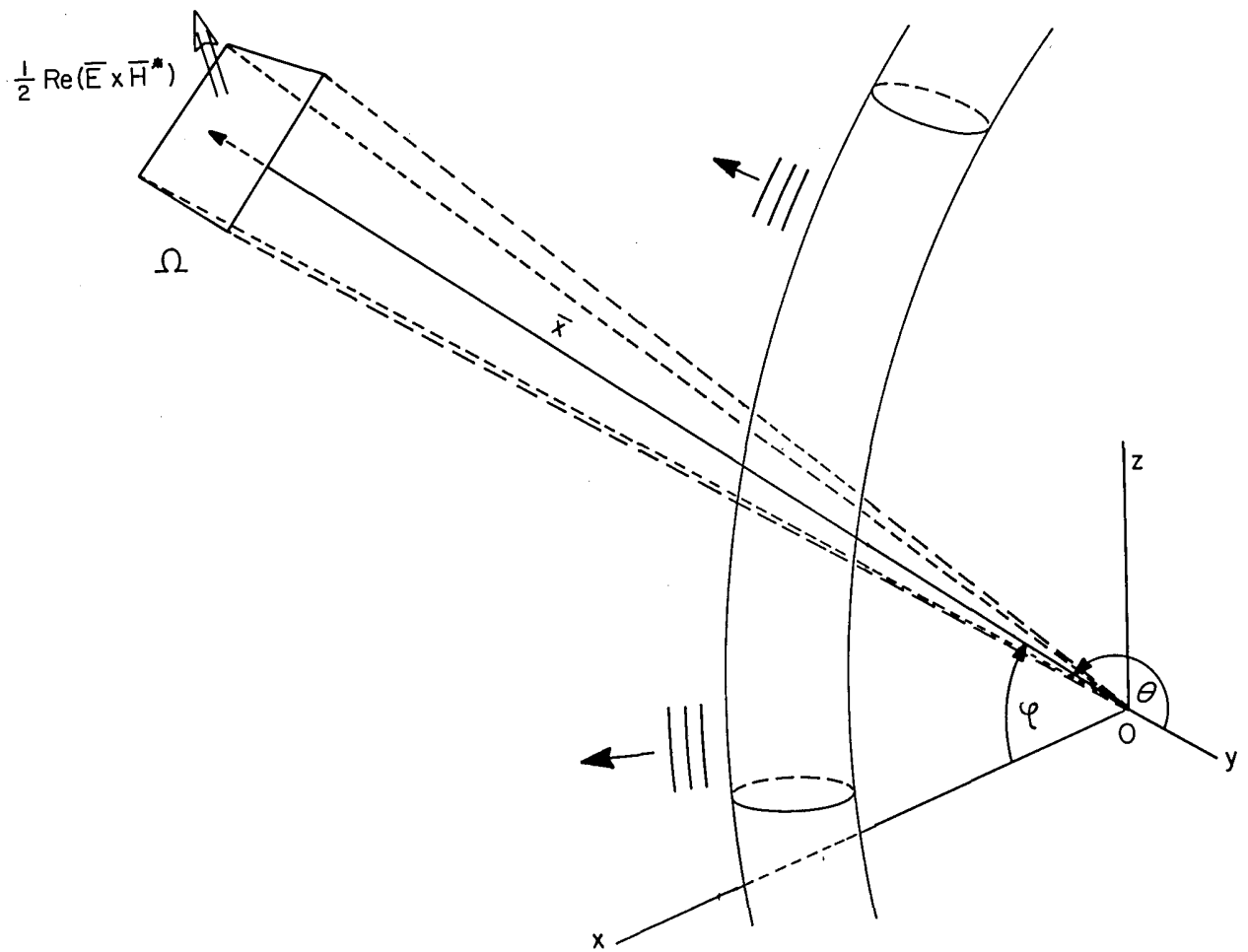

Fig. 5. Flow of radiated energy from a bent waveguide across a finite solid angle $\Omega$ on a spherical surface of very large radius $|\bar{x}|$ ( $\gg R$ ), the center of which is at the origin 0 . The solid angle is determined by $\pi / 2-\theta_{0}<\theta<\pi / 2+\theta_{0}, \varphi_{1}<\varphi<\varphi_{2}$ (see the text). The double-line arrow $(\Rightarrow$ ) represents the outgoing time-averaged Poynting vector $1 / 2 \operatorname{Re}\left(\bar{E} \times \bar{H}^{*}\right)$. 
By using expressions (10) and (18) and Eqs. (15), (16), (19), and $(20), F_{\text {rad }}$ becomes

$$
\begin{aligned}
F_{\mathrm{rad}} \sim & (R / 16 \pi) \int_{T} \mathrm{~d} x_{1}{ }^{\prime} \mathrm{d} y^{\prime} \int_{T} \mathrm{~d} x_{1}{ }^{\prime \prime} \mathrm{d} y^{\prime \prime} k^{4}\left[\epsilon\left(x_{1}{ }^{\prime}, y^{\prime}\right)-\epsilon_{c}\right] \\
& \times\left[\epsilon\left(x_{1}{ }^{\prime \prime}, y^{\prime \prime}\right)-\epsilon_{c}\right] \sum_{\alpha_{1} \alpha^{1}=0}^{N} \bar{A}_{\alpha \alpha^{\prime}} \operatorname{Re}\left[\bar{E}_{0 \alpha}\left(x_{1}^{\prime}, y^{\prime}\right)\right. \\
& \left.\times \bar{H}_{0 \alpha^{\prime}}\left(x_{1}^{\prime \prime}, y^{\prime \prime}\right)\right] c_{\alpha} c_{\alpha}{ }^{*}
\end{aligned}
$$

with

$$
\begin{aligned}
\bar{A}_{\alpha \alpha^{\prime}}= & \int_{\varphi_{1}}^{\varphi_{2}} \mathrm{~d} \varphi \sin \theta \mathrm{d} \theta\left[\gamma_{1 \alpha}(\theta) \gamma_{1 \alpha^{\prime}}(\theta)\right]^{-1 / 2} \\
& \times \exp \left\{R\left[\beta_{\alpha} \Lambda_{\alpha}(\theta)+\beta_{\alpha^{\prime}} \Lambda_{\alpha^{\prime}}(\theta)\right]\right\} \\
& \times \exp \left[\gamma_{1 \alpha}(\theta) x_{1}{ }^{\prime}+\gamma_{1 \alpha^{\prime}}(\theta) x_{1}{ }^{\prime \prime}(\bar{x} /|\bar{x}|) .\right.
\end{aligned}
$$

A glance at Eqs. (14) and (23) indicates that the largest contribution to the $\theta$ integration comes from the neighborhood of $\theta=\pi / 2$ and is due to the factor $\exp \left\{R\left[\beta_{\alpha} \Lambda_{\alpha}(\theta)+\right.\right.$ $\left.\beta_{\alpha^{\prime}} \Lambda_{\alpha^{\prime}}(\theta)\right]$, since $R$ is large. Therefore not much precision is lost by setting $\theta=\pi / 2$ in all factors (except $\exp \left\{R\left[\beta_{\alpha} \Lambda_{\alpha}(\theta)+\right.\right.$ $\left.\left.\left.\beta_{\alpha^{\prime}} \Lambda_{\alpha^{\prime}}(\theta)\right]\right\}\right)$ in the right-hand side of Eq. (23) and then extending the $\theta$ integration from $\theta=0$ up to $\theta=\pi$ (that is, at this stage, $\left.\theta_{0}=\pi / 2\right)$. Thus, since $\gamma_{1 \alpha}(\theta=\pi / 2)=\gamma_{\alpha}$,

$$
\bar{A}_{\alpha \alpha^{\prime}} \sim 2\left(\gamma_{\alpha} \gamma_{\alpha^{\prime}}\right)^{-1 / 2} \exp \left(\gamma_{\alpha} x_{1}^{\prime}-\gamma_{\alpha^{\prime}} x_{1}{ }^{\prime \prime}\right) A_{1_{\alpha \alpha}} \bar{A}_{2},
$$

with

$$
\begin{aligned}
A_{1_{\alpha \alpha^{\prime}}} & =\int_{0}^{\pi / 2} \mathrm{~d} \theta \exp \left\{R\left[\beta_{\alpha^{\prime}} \Lambda_{\alpha}(\theta)+\beta_{\alpha^{\prime}} \Lambda_{\alpha^{\prime}}(\theta)\right]\right\} \\
& \simeq\left[\pi / R\left(\gamma_{\alpha}+\gamma_{\alpha^{\prime}}\right)\right]^{1 / 2} \exp \left\{R\left[\beta_{\alpha} \Lambda_{\alpha}(\pi / 2)+\beta_{\alpha^{\prime}} \Lambda_{\alpha^{\prime}}(\pi / 2)\right]\right\}
\end{aligned}
$$

and

$$
\bar{A}_{2}=\int_{\varphi_{1}}^{\varphi_{2}} \mathrm{~d} \varphi(\bar{x} /|\bar{x}|) .
$$

$A_{1_{\alpha \alpha^{\prime}}}$ has been evaluated by using Laplace's method, ${ }^{20}$ which was allowed since $R$ is large.

In what follows we shall restrict ourselves to the case in which only one mode propagates initially for $z>z_{0}$, that is, $c_{\alpha_{0}}=1, c_{\alpha}=0$ for $\alpha \neq \alpha_{0}$ (and we omit, then, the subscript $\left.\alpha_{0}\right)$, in order to define the bending-loss coefficient for such a propagation mode. Notice that $\bar{A}_{2}$ in Eq. (26) is independent of $\alpha, \alpha^{\prime}$; an approximate and more manageable expression for it, which is also valid for the multimode situation, will be given below.

To obtain a manageable expression for the bending-loss coefficient for purposes of numerical estimates, we shall consider further approximations here. We shall suppose that the incoming averaged Poynting vector is almost parallel to the $z$ axis, which is equivalent to accepting that the energy lost by the waveguide comes solely from the existence of bending. regions. The largest contribution to $F_{\text {rad }}$ can then be expected from $\varphi-$ values close to $\pi / 2$, so that one may reasonably set $\varphi_{1}=\pi / 2-\varphi_{0}, \varphi_{2}=\pi / 2+\varphi_{0}, \varphi_{0}$ being small, and $\bar{A}_{2} \simeq 2 \varphi_{0} \bar{u}_{z}$. By collecting these results, we get

$$
F_{\mathrm{rad}} \simeq R^{1 / 2} \exp [2 \beta R \Lambda(\pi / 2)] 2 \varphi_{0} F,
$$

with

$$
\begin{aligned}
F= & {\left[1 / 8\left(2 \pi \gamma^{3}\right)^{1 / 2}\right] \int_{T} \mathrm{~d} x_{1}{ }^{\prime} \mathrm{d} y^{\prime} \int_{T} \mathrm{~d} x_{1}{ }^{\prime \prime} \mathrm{d} y^{\prime \prime} k^{4}\left[\epsilon\left(x_{1}{ }^{\prime}, y^{\prime}\right)-\epsilon_{c}\right] } \\
& \times\left[\epsilon\left(x_{1}^{\prime \prime}, y^{\prime \prime}\right)-\epsilon_{c}\right] \exp \left[\gamma\left(x_{1}{ }^{\prime}-x_{1}{ }^{\prime \prime}\right)\right] \\
& \times\left\{\bar{u}_{z} \operatorname{Re}\left[\bar{E}_{0}\left(x_{1}{ }^{\prime}, y^{\prime}\right) \times \bar{H}_{0}\left(x_{1}^{\prime \prime}, y^{\prime \prime}\right)\right]\right\} .
\end{aligned}
$$

The ratio $F_{\text {rad }} / 2 \varphi_{0} R$ can be interpreted as the energy flux radiated per unit length of the waveguide. We then define the bending loss coefficient $\tau$ as

$$
\begin{aligned}
\tau & =\left(1 / F_{\text {in }}\right)\left(F_{\text {rad }} / 2 \varphi_{0} R\right) \\
& =\frac{\exp \left\{-2 R \beta\left[\tanh ^{-1}\left(\beta^{-1} \gamma\right)-\beta^{-1} \gamma\right]\right\}}{R^{1 / 2}} \frac{F}{F_{\text {in }}},
\end{aligned}
$$

which is normalized to the incoming flux.

\section{NUMERICAL ESTIMATES AND COMPARISON WITH OTHER PREVIOUS RESULTS}

We are interested in analyzing numerically the main features appearing in the bending-loss coefficient obtained in the present study and those presented earlier. The one defined by Marcuse ${ }^{19}$ for a homogeneous two-dimensional slab of half-width' $d$ is

$$
\tau_{\mathrm{M}}=\frac{\gamma^{2}}{\beta(1+\gamma d)} \frac{\left(\epsilon-\epsilon_{c}\right) k^{2}-\gamma^{2}}{\left(\epsilon-\epsilon_{c}\right) k^{2}} \exp (2 \gamma d) \exp \left[-2 / 3\left(\gamma^{3} / \beta^{2}\right) R\right],
$$

and

$$
\begin{aligned}
\tau_{\mathrm{SL}}= & \left(\pi V^{8} / 16 \rho R W^{3}\right) \exp \left[-4 / 3(R / \rho)\left(\Delta W^{3} / V^{2}\right)\right] \\
& \times\left[\int_{0}^{\infty}\{1-f(r)\} F_{0}(r) r \mathrm{~d} r\right] / \int_{0}^{\infty} F_{0}{ }^{2}(r) r \mathrm{~d} r
\end{aligned}
$$

has been defined by Snyder and Love as the power-attenuation coefficient for a homogeneous three-dimensional fiber of circular cross section and radius $\rho$, where $\left(r=|\mathbf{x}|, \epsilon=n^{2}\right)$

$$
\begin{aligned}
n^{2}(r) & =n_{c o}{ }^{2}[1-2 \Delta f(r)], \quad \Delta=\left(n_{c o}{ }^{2}-n_{c l}{ }^{2}\right) / 2 n_{c o}{ }^{2}, \\
V & =k \rho\left(n_{c o}{ }^{2}-n_{c l}{ }^{2}\right), \quad W=\rho\left(\beta^{2}-k^{2} n_{c l}{ }^{2}\right)^{1 / 2},
\end{aligned}
$$

with $k n_{c l}<\beta<k n_{c o}$ and $F_{0}(r)$ as the radial function characterizing the propagation mode. The order of magnitude and main variations in Eqs. (29)-(31) are determined by the exponential factors.

We have studied, for the same three-dimensional fiber of circular cross section characterized above, the following dimensionless coefficients:

$$
\xi_{M}=2 / 3\left(\gamma^{3} / \beta^{2}\right) R
$$

and

$$
\xi_{S}=4 / 3(R / \rho)\left(\Delta W^{3} / V^{2}\right),
$$

in comparison with

$$
\xi_{A}=2 \beta R\left[\tanh ^{-1}(\gamma / \beta)-(\gamma / \beta)\right],
$$




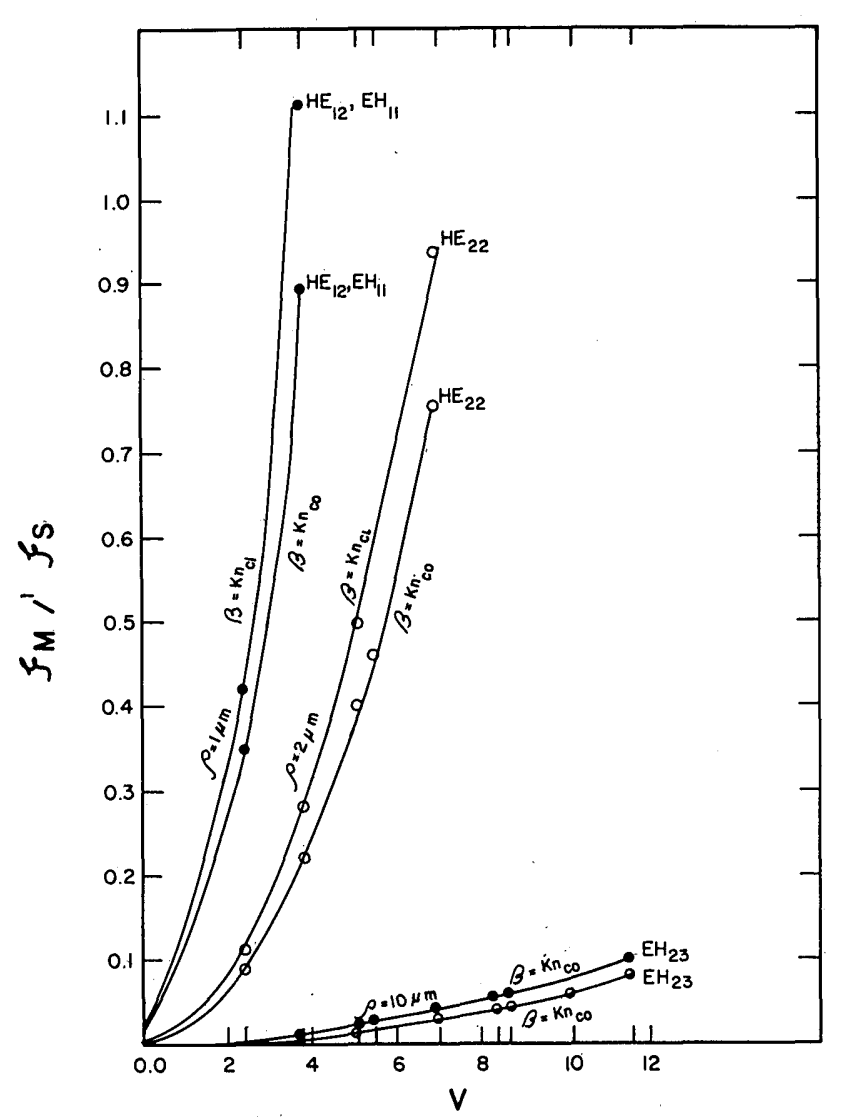

Fig. 6. Behavior of the ratio $\xi_{M} / \xi_{S}$ at the upper bound $\left(\beta=k n_{0}\right)$ and lower bound $\left(\beta=k n_{c}\right)$ versus the $V$ parameter. Three values for the radius $\rho$ of the fiber have been considered: $1.0,2.0$, and $10.0 \mu \mathrm{m}$, covering all the possibilities from a monomode to a multimode regime. Other parameters are $R=1.0 \mathrm{~cm}, \lambda=0.9 \mu \mathrm{m}$, and $\Delta=0.06$.

which has been introduced in the present study [Eq. (29)] and also coincides with the one appearing in Marcuse's twodimensional treatment (when no approximations are considered). Notice that by expanding $\tanh ^{-1}(\gamma / \beta)$ into powers, $\left.\xi_{A}=2 \beta R \cdot(1 / 3)(\gamma / \beta)^{3}+(1 / 5)(\gamma / \beta)^{5}+\ldots\right]$, so that the lowest nontrivial approximation for $\xi_{A}$ is $\xi_{M}$. First, we have studied $\left(\xi_{A} / \xi_{M}\right)-1$ for the $\alpha$ th propagation mode $(\alpha=0,1, \ldots)$. One has

$$
\left(\xi_{A} / \xi_{M}\right)-1 \simeq 3 / 5\left(W_{\alpha} / \rho \beta_{\alpha}\right)^{2}=3 / 5\left[1-\left(k n_{c l} / \beta_{\alpha}\right)^{2}\right] .
$$

For a fixed core radius $\rho$ and a fixed relative index difference $\Delta$, the values are independent of the modal parameter $V$. For $\rho=1 \mu \mathrm{m}$ and $\Delta=0.013,\left(\xi_{A} / \xi_{M}\right)-1=0.01$, and for $\rho=10$ $\mu \mathrm{m}$ and $\Delta=0.06,\left(\xi_{A} / \xi_{M}\right)-1=0.08$. The approximation $\xi_{A}$ $\simeq \xi_{M}$ is then a good one for at least up to 2 orders of magnitude.

We are particularly interested in the numerical analysis of the following:

$$
\xi_{M} / \xi_{S}=\left(V_{\alpha} / \rho \beta_{\alpha}\right)^{2}(1 / 2 \Delta)
$$

with $\alpha=0,1,2, \ldots$,

$$
g=\exp \left\{\xi_{S}\left[\left(\xi_{M} / \xi_{S}\right)-1\right]\right\},
$$

which would give the main variation in the ratio $\tau_{S L} / \tau$ and the difference

$$
\xi_{M}-\xi_{S}=\xi_{S}\left[\left(\xi_{M} / \xi_{S}\right)-1\right]=\ln g .
$$

For a fixed incoming wavelength $(\lambda=0.9 \mu \mathrm{m})$ and fixed $\Delta=$ $0.06\left(\epsilon_{c o} / \epsilon_{c l} \sim 1.1\right)$, the ratio $\xi_{M} / \xi_{S}$ given in Eq. (36) is displayed in Fig. 6 as a function of the $V$ parameter. Its values depend on the behavior of the waveguide as multimode (for $\rho$ $=10 \mu \mathrm{m}$, the waveguide supports 18 modes with the above physical parameters) or quasi-monomode ( $\rho=1 \mu \mathrm{m}$, with 5 modes: $\mathrm{TE}_{01}, \mathrm{TM}_{01}, \mathrm{HE}_{11}, \mathrm{HE}_{12}, \mathrm{EH}_{11}$ ) behavior. The ratio $\xi_{M} / \xi_{S}$ has a higher dispersion in values as the waveguide approaches an almost monomode behavior, reaching a value of 1.1 for the $\mathrm{HE}_{12}$ and $\mathrm{EH}_{11}$ modes. This dispersion drastically decreases as the waveguide propagates light under a multimode behavior. In this case, $\xi_{M} \leqslant 0.1 \xi_{S}$, which goes up to 1 order of magnitude for the $\mathrm{EH}_{23}$ mode. Both coefficients, $\xi_{M}$ and $\xi_{S}$, almost coincide in the boundary of the near-cutoff condition approximation $\beta=k n_{\text {co }}$, provided that the waveguide be almost monomode.

We have also checked numerically Eqs. (37) and (38). The results are displayed in Fig. 7. In this case only the bound for $\beta=k n_{c o}$ is displayed, as the obtained values for $\beta$ $=k n_{c l}$ are almost negligible. We observe here a big dispersion in the numerical factors as the waveguide supports a higher number of modes. The maximum dispersion appears for $\rho=10 \mu \mathrm{m}$ for both the $g$ function (left axis) and the $\ln g$ function (right axis). For the $g$ function only values from $V$ $=7.016$ are displayed, because for smaller $V$ it reaches values of the order of $10^{-16}$ and even less. It is clear that here the discrepancy is very critical, as it goes up to several orders of magnitude. The discrepancy is less important for an almost monomode waveguide, for which the ratio $g$ varies only 1 order of magnitude.

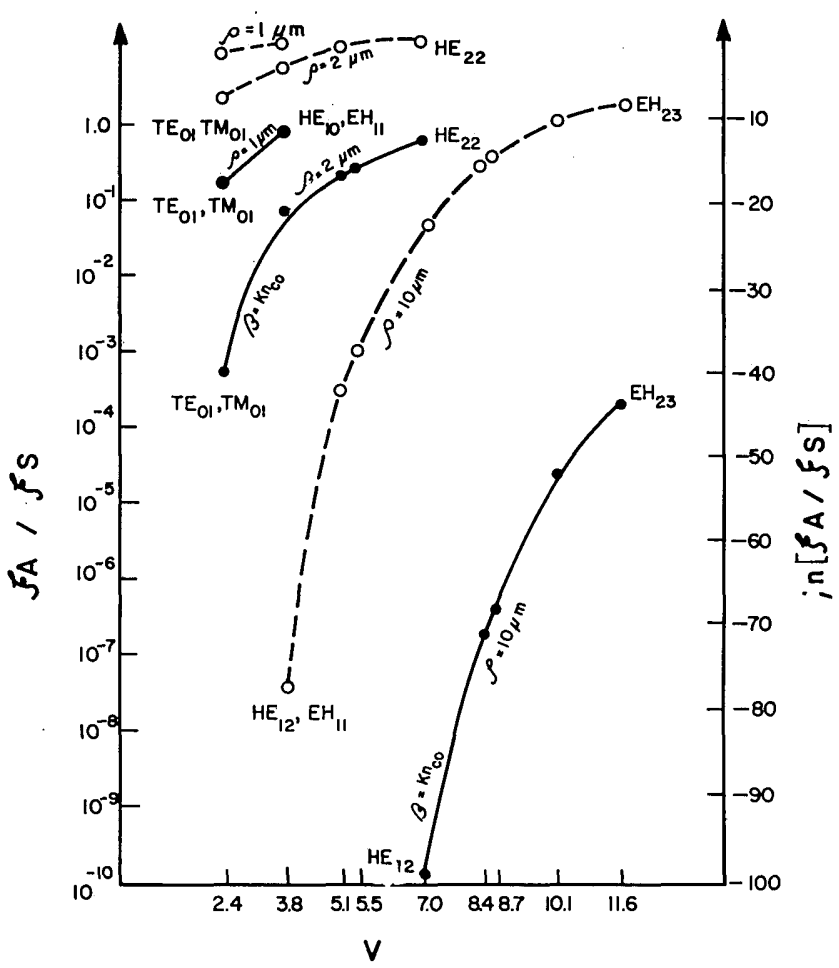

Fig. 7. Behavior of the ratios $\xi_{A} / \xi_{S}$ (solid lines) and $\ln \xi_{A} / \xi_{S}$ (dashed lines) versus the $V$ parameter at the upper bound $\beta=k n_{0}$. Three values for the radius $\rho$ of the fiber have been considered: 1.0 $\mu \mathrm{m}$ (almost monomode), $2.0 \mu \mathrm{m}$ (intermediate regime), and $10.0 \mu \mathrm{m}$ (multimode); a fixed radius of curvature $R=1.0 \mathrm{~cm}$ was used. 


\section{DISCUSSION}

To go deeper into the above results, it is important to discuss some basic differences between Marcuse's two-dimensional method $^{15}$ and our new three-dimensional one:

(1) Marcuse considered explicit solutions (up to an unknown amplitude) for two-dimensional step-index bent waveguides without any reference to Green's functions, whereas our use of the latter enables us to treat waveguides with arbitrary refractive-index profiles.

(2) Before obtaining a fully explicit formula for the optical field far from the waveguide, that is, for $|\mathbf{x}| \gg R[\mathbf{x}=(x, y)$ in two dimensions], Marcuse was forced to formulate a "matching condition" for $\mathbf{x}$ outside, but near, the fiber: there, his solutions for the two-dimensional bent waveguide had to coincide (for large $R$ ) with a certain solution for perfectly straight step-index fiber, which enabled him to obtain the unknown amplitude referred to above. We emphasize the deep significance of such a matching condition and refer to Marcuse's treatment ${ }^{19}$ for the details. On the other hand, in order to obtain the general formulas in expression (10) and Eqs. (15) and (16), we did not need to impose any matching condition: our asymptotic optical field followed from the integral Eq. (5).

(3) In the formulation of his matching condition, Marcuse used an asymptotic formula for an outgoing Hankel function with large order and argument, whereas we are led to approximate a Bessel function.

(4) The most delicate aspect in the derivation of expression (10) and Eqs. (15) and (16) from Eq. (5) was to find a suitable approximation for $J_{\beta R}\left[k \epsilon_{c}{ }^{1 / 2} \sin \theta\left(x^{\prime 2}+z^{\prime 2}\right)^{1 / 2}\right]$. In this connection, one may ask whether expression (13) actually suffices and whether higher-order corrections in the equation (or even a different form) would be required. In Appendix B, we shall outline a consistency argument that indicates that the approximation in expression (13) suffices. As will be seen there, such a consistency check could be regarded as a general (Green's function) version, for three dimensions, of Marcuse's matching condition in two dimensions.

(5) The specific contribution coming from our threedimensional analysis is indicated by the $1 / \sqrt{R}$ coefficient, which coincides with the one presented in Ref. 20. This coefficient does not appear in Marcuse's treatment, whereas $\tanh ^{-1}\left[\left(\beta^{-1} \gamma\right)-\beta^{-1} \gamma\right]$ does appear in his bending-loss coefficient. Therefore our analysis is a generalization of both of these formulations.

(6) The numerical analysis shows that there exists a discrepancy between $\xi_{S}$ and $\xi_{M}$ (or $\xi_{A}$ ). The ratios between these coefficients vary as the waveguide changes in behavior as monomode or multimode. As the correction to the third degree of approximation in the general formula is small and independent of $V$, we conclude that using the $\tau_{A}$ appears to be less compromised. The differences between $\xi_{A}\left(\xi_{M}\right)$ and $\xi_{S}$ (1) are rather small for monomode behavior near cutoff, (2) increase as the behavior of the waveguide changes from monomode to multimode, and (3) decrease as the parameter $V$ increases for a given core radius and propagation mode.

(7) The effect of field deformation that is due to bending is also important, and it has been considered for particular (although important) cases by Marcuse ${ }^{9}$ and by Sakai and Kimura. ${ }^{7}$ It does not seem to be easy to compare our meth- od with Marcuse's approach to the field deformation effect. On the other hand, according to Sakai and Kimura, such an effect amounts to certain specific corrections of order $R^{-1}$ relative to the leading contribution. Accordingly, the effect of field deformation does not appear to be incorporated into expression (10) and Eqs. (15) and (16). Our method may still provide some basis for conjecturing how the effect of field deformation that is due to bending could be taken into account. For instance, this could be done, at least partially, by keeping terms of order $R^{-1}$ on the right-hand side of (11) relative to the leading contribution, as given by expression (10) and Eqs. (15) and (16): such terms could arise as corrections to expression (A1) [compare with Eq. (9.3.8) of Ref. 23]. However, there could be additional $R^{-1}$ corrections arising, for instance, from the approximations leading from Eq. (5) to expression (10) and Eq. (11): they would require an elaborate treatment, which lies beyond the scope of this work.

\section{APPENDIX A}

We shall outline the derivation of expression (13). We start by using the following asymptotic representation [see Eq. (9.3.2) of Ref. 23]:

$$
\begin{aligned}
& J_{\beta R}\left[k \epsilon_{c}{ }^{1 / 2} \sin \left(x^{\prime 2}+z^{\prime 2}\right)^{1 / 2}\right] \simeq 1 /\left\{[2 \pi \beta R \tanh \alpha]^{1 / 2}\right\} \\
&\times \exp [\beta R(\tanh \alpha-\alpha)]\}, \\
& \cosh \alpha=\beta R / k \epsilon_{c}{ }^{1 / 2} \sin \theta\left(x^{\prime 2}+{z^{\prime}}^{\prime 2}\right)^{1 / 2} \\
& \simeq\left(\beta / k \epsilon_{c}{ }^{1 / 2} \sin \theta\right)\left[1-\left(x_{1}{ }^{\prime} / R\right)\right]
\end{aligned}
$$

as $\left(x^{\prime 2}+z^{\prime 2}\right)^{-1 / 2} \simeq R^{-1}\left[1-\left(x_{1}{ }^{\prime} / R\right)\right]$, since $x_{1}{ }^{\prime}$ has a limited variation and $R$ is, by assumption, suitably large so that $\left|x_{1}\right|$ $\ll R$ holds [recall approximation (1) after expression (9)]. Thus both the order and the argument in $J_{\beta R}\left[k \epsilon_{c}{ }^{1 / 2} \sin \theta\left(x^{\prime 2}\right.\right.$ $\left.\left.+z^{\prime 2}\right)^{1 / 2}\right]$ are large, and the former is larger than the latter. Consequently, since $\beta>k \epsilon_{c}{ }^{1 / 2}$ [recall the last Eq. (2)], one has $\cosh \alpha>1$ for any $x^{\prime}$, which is integrated over in Eq. (11), and so the validity of expression (A1) is justified.

Next, we shall give more suitable approximations for the right-hand side of expression (A1). In its denominator, it suffices to set, if $v=\tanh \alpha$,

$$
\begin{aligned}
\beta R v & =\beta R\left[(\cosh \alpha)^{2}-1\right]^{1 / 2} / \cosh \alpha \\
& =(\beta R)^{2}-k^{2} \epsilon_{c} \sin \theta\left(x^{\prime 2}+{z^{\prime}}^{2}\right)^{1 / 2} \\
& \simeq R \gamma_{1}(\theta) .
\end{aligned}
$$

In order to deal with the exponential in expression (A1), the following calculations and approximations, which generalize those made by Marcuse in two dimensions, ${ }^{19}$ will be useful:

$$
\begin{aligned}
\alpha-\tanh \alpha & =\sum_{n=3}^{\infty}(v)^{n} / n \\
(v)^{n} & \simeq\left[\gamma_{1}(\theta) / \beta\right]^{n}\left[1-\gamma_{1}(\theta)\left(2 x_{1}^{\prime} / R\right)\right]^{n / 2} \\
& \simeq\left[\gamma_{1}(\theta) / \beta\right]^{n}\left[1-n \sigma(\theta)\left(x_{1}^{\prime} / R\right)\right] \\
\sigma(\theta) & =k^{2} \epsilon_{c} \sin \theta /\left[\gamma_{1}(\theta)\right]^{2} .
\end{aligned}
$$

On combining Eq. (A4) and expression (A5), 


$$
\begin{aligned}
\alpha-\tanh \alpha \simeq & \sum_{n=3}^{\infty}(1 / n)\left[\gamma_{1}(\theta) / \beta\right]^{n}-\sigma(\theta)\left(x_{1}{ }^{\prime} / R\right) \sum_{n=3}^{\infty}\left[\gamma_{1}(\theta) / \beta\right]^{n} \\
= & \tanh ^{-1}\left[\gamma_{1}(\theta) / \beta\right]-\left[\gamma_{1}(\theta) / \beta\right] \\
& -\sigma(\theta)\left(x_{1}{ }^{\prime} / R\right)\left[\gamma_{1}(\theta) / \beta\right]^{3} /\left\{1-\left[\gamma_{1}(\theta) / \beta\right]^{2}\right\} . \quad \text { (A7) }
\end{aligned}
$$

Finally, the combined use of expressions (A1)-(A3) and (A7) together with

$$
\beta \sigma(\theta)\left[\gamma_{1}(\theta) / \beta\right]^{3} /\left\{1-\left[\gamma_{1}(\theta) / \beta\right]^{2}\right\}=\gamma_{1}(\theta)
$$

leads directly to expression (13).

\section{APPENDIX B}

We shall outline a nontrivial check of consistency of our basic approximations. Specifically, we shall prove that, by using asymptotic formulas similar to expression (13), Eq. (5) becomes Eq. (3) when $\bar{x}$ lies in certain regions (see below).

The following alternative representation for $G\left(\bar{x}-\bar{x}^{\prime}\right)$ will be necessary:

$$
\begin{aligned}
G\left(\bar{x}-\bar{x}^{\prime}\right)= & (1 / 4 \pi) \int \mathrm{d} l_{y} \exp \left[i l_{y}\left(y-y^{\prime}\right)\right] \\
& \times(-1 / 4 i) \sum_{M=-\infty}^{+\infty} \exp \left[i M\left(\varphi-\varphi^{\prime}\right)\right] \\
& \times H_{|M|}^{(1)}\left[\left(k^{2} \epsilon_{c}-l_{y}^{2}\right)^{1 / 2} r_{>}\right] \\
& \times J_{|M|}\left[\left(k^{2} \epsilon_{c}-l_{y}^{2}\right)^{1 / 2} r_{<}\right] .
\end{aligned}
$$

In Eq. (B1), $\left.H_{|M|}\right|^{(1)}$ is the standard Hankel outgoing function of order $M, r_{<}$and $r>$ are the smallest and the largest of $\left(x^{2}+z^{2}\right)^{1 / 2}$ and $\left(x^{\prime 2}+z^{\prime 2}\right)^{1 / 2}$, and $\varphi$ and $\varphi^{\prime}$ are the angles formed by two-dimensional vectors $(x, z)$ and $\left(x^{\prime}, z^{\prime}\right)$ with the $x$ axis. Equation (B1) can be obtained trivially from Eq. (6) by recalling Eq. (4) and the standard partial-wave expansion for $H_{0}^{(1)}$ in the $x z$ plane (compare with Ref. 23). For brevity we omit details. We next consider Eq. (5), replace $G\left(\bar{x}-\bar{x}^{\prime}\right)$ by the right-hand side of Eq. (B1) [expressions (7) and (9) are not used here!], use the approximations (1)-(3) formulated after expression (9) and, furthermore, assume that

(4) $\bar{x}$ lies outside but near the waveguide, with $\theta$ close to $\pi / 2$ and $|\bar{x}|>R$ (but $|\bar{x}| \gg R$ is not supposed here!), and, more specifically, that $|\bar{x}| \simeq\left(x^{2}+z^{2}\right)^{1 / 2}=r_{>} \simeq R+x_{1}, 0<x_{1} \ll R$, $x_{1}-x_{1}{ }^{\prime}>0$, and $\varphi$ is small so that $z \simeq R \varphi$. A direct calculation analogous to the one yielding expression (10) and Eq. (11) then leads from Eqs. (5) and (B1) to

$$
\begin{aligned}
\bar{E}(\bar{x}) \simeq & \exp (i \beta R \varphi) \int_{T} \mathrm{~d} x_{1}^{\prime} \mathrm{d} y^{\prime} Q k^{2}\left[\epsilon\left(x_{1}^{\prime}, y^{\prime}\right)-\epsilon_{c}\right] \bar{E}_{0}\left(x_{1}^{\prime}, y^{\prime}\right), \\
Q= & (-1 / 2 i) R \int \frac{\mathrm{d} l_{y}}{2 \pi} \exp \left[i l_{y}\left(y-y^{\prime}\right)\right] \\
& \times H_{\beta R}^{(1)}\left[\left(k^{2} \epsilon_{c}-l_{y}^{2}\right)^{1 / 2}\left(x^{2}+z^{2}\right)^{1 / 2}\right] \\
& \times J_{\beta R}\left[\left(k^{2} \epsilon_{c}-l_{y}^{2}\right)^{1 / 2}\left(x^{\prime 2}+z^{\prime 2}\right)^{1 / 2}\right] .
\end{aligned}
$$

The interest of letting $\bar{x}$ lie in the region assumed in assumption (4) above should now be apparent: since $R$ is large, curvature effects should not be appreciable, and the actual propagation mode in the bent waveguide should coincide with that for the perfectly straight one. Consistent with such an intuition, for such $\bar{x}$ values the approximation $\bar{E}(\bar{x}) \sim \exp (i \beta z) \bar{E}_{0}\left(x_{1} y\right), z \simeq R \varphi$ would be valid at the lefthand side of expression (B2) and the latter, when $\exp (i \beta R \varphi)$ is factored out, would become identical to Eq. (3), if it were true that, under all the above approximations,

$$
Q \sim(-1 / 4 i) H_{0}{ }^{(1)}\left\{i \gamma\left[\left(x_{1}-x_{1}{ }^{\prime}\right)^{2}+\left(y-y^{\prime}\right)^{2}\right]^{1 / 2}\right\}
$$

holds. Notice that since the right-hand side of expression (B4) does not depend on $R$, its validity would require the cancellation of all curvature effects in $Q$. We shall prove that expression (B4) is certainly valid.

We shall approximate the Bessel function contributing to the right-hand side of Eq. (B3) in a way similar to Eq. (11) and expression (13), as the former also has large order and argument:

$$
\begin{aligned}
J_{\beta R}\left[\left(k^{2} \epsilon_{c}-l_{y}^{2}\right)^{1 / 2}\left(x^{2}+{z^{\prime}}^{2}\right)^{1 / 2}\right] \simeq & {[1 /(2 \pi \beta R \tanh \alpha)] } \\
& \times \exp [\beta R(\tanh \alpha-\alpha)],
\end{aligned}
$$

$$
\begin{aligned}
\cosh \alpha & =\frac{\beta R}{\left(k^{2} \epsilon_{c}-l_{y}^{2}\right)^{1 / 2}}\left(x^{\prime 2}+{z^{\prime}}^{2}\right)^{1 / 2} \\
& \simeq\left[\beta /\left(k^{2} \epsilon_{c}-l_{y}^{2}\right)^{1 / 2}\right]\left[1-\left(x_{1}{ }^{\prime} / R\right)\right] .
\end{aligned}
$$

One could be worried about the validity of the approximation in expression (B5) for values of $l_{y}$ such that $k^{2} \epsilon_{c}-l_{y}{ }^{2}<0$, since the condition cosh $\alpha>1$ would then no longer hold. Such a difficulty disappears for the following reasons:

(a) The largest contribution to the integral in Eq. (B3) can be expected to come from $l_{y}$ values such that $k^{2} \epsilon_{c}-l_{y}{ }^{2}>$ 0 , since for $l_{y}^{2} \gg k^{2} \epsilon_{c}$ the product $H_{\beta R}{ }^{(1)} J_{\beta R}$ is exponentially damped [as $\left.\left(x^{2}+z^{2}\right)^{1 / 2}>\left(x^{\prime 2}+z^{\prime 2}\right)^{1 / 2}\right]$.

(b) The troublesome $\left(k^{2} \epsilon_{c}-l_{y}^{2}\right)^{1 / 2}$ will be canceled out automatically at certain stages in the following calculations, after which it will no longer appear; so, beyond those stages, it will also become obvious that the $l_{y}$ integration can be extended from $-\infty$ up to $+\infty$.

Calculations similar to those in Appendix A, with $\sin ^{2} \theta k^{2} \epsilon_{c}$ replaced by $k^{2} \epsilon_{c}-l_{y}^{2}$, yield (with $\gamma^{2}=\beta^{2}-k^{2} \epsilon_{c}$ )

$$
\begin{array}{rc}
R \tanh \alpha \simeq R \gamma_{2} ; \quad \gamma_{2}=\left(\gamma^{2}+l_{y}^{2}\right)^{1 / 2}, \\
\sigma_{1}=\left(k^{2} \epsilon_{c}-l_{y}^{2}\right) / \gamma_{2}^{2}, \\
\alpha-\tanh \alpha \simeq \tanh ^{-1}\left[\gamma_{2} / \beta\right]-\left[\gamma_{2} / \beta\right] \\
-\sigma_{1}\left(x_{1}^{\prime} / R\right)\left[\left(\gamma_{2} / \beta\right)^{3}\right] /\left[1-(\gamma / \beta)^{2}\right] .
\end{array}
$$

Simple algebra shows that

$$
\sigma_{1} \beta\left(\gamma_{2} / \beta\right)^{3} /\left[1-\left(\gamma_{2} / \beta\right)^{2}\right]=\gamma_{2} .
$$

The right-hand side of expression (B7b), when Eq. (B8) is used, and that of the last expression (B7a) no longer contain the troublesome $\left(k^{2} \epsilon_{c}-l_{y}^{2}\right)^{1 / 2}$. When the above results are combined, expression (B5) becomes 


$$
\begin{aligned}
J_{\beta R}\left[\left(k^{2} \epsilon_{\mathrm{c}}-l_{y}{ }^{2}\right)^{1 / 2}\left(x^{\prime 2}+z^{\prime 2}\right)^{1 / 2}\right] \simeq & \left(\operatorname { e x p } \left\{\beta R \left[\left(\gamma_{2} / \beta\right)\right.\right.\right. \\
- & \left.\left.\left.\tanh ^{-1}\left(\gamma_{2} / \beta\right)\right]\right\}\right) / \\
& \left\{\left[\left(2 \pi R \gamma_{2}\right)^{1 / 2}\right] \exp \left(\gamma_{2} x_{1}{ }^{\prime}\right)\right\}
\end{aligned}
$$

In a similar way, we shall approximate the outgoing Hankel function appearing in the right-hand side of Eq. (B3) as (see, for instance, Ref. 23)

$$
\begin{gathered}
H_{\beta R}{ }^{(1)}\left[\left(k^{2} \epsilon_{c}-l_{y}{ }^{2}\right)^{1 / 2}\left(x^{2}+z^{2}\right)^{1 / 2}\right] \simeq-i \exp [\beta R(\alpha-\tanh \alpha)] / \\
{[(\pi / 2) \beta R \tanh \alpha]^{1 / 2},}
\end{gathered}
$$

$$
\begin{aligned}
\cosh \alpha & =[\beta R] /\left[\left(k^{2} \epsilon_{c}-l_{y}{ }^{2}\right)^{1 / 2}\left(x^{2}+z^{2}\right)^{1 / 2}\right. \\
& \simeq\left(\beta /\left[\left(k^{2} \epsilon_{c}-l_{y}{ }^{2}\right)^{1 / 2}\right]\left[1+\left(x_{1} / R\right)\right] .\right.
\end{aligned}
$$

The calculations leading to expression (B9) also hold for $H_{\beta R}{ }^{(1)}$, provided that $x_{1}{ }^{\prime}$ be replaced by $x_{1}$; consequently,

$$
\begin{aligned}
H_{\beta R}{ }^{(1)}\left[\left(k^{2} \epsilon_{c}-l_{y}{ }^{2}\right)^{1 / 2}\left(x^{2}+z^{2}\right)^{1 / 2}\right] \simeq & -i \exp \left\{\beta R \left[\tanh ^{-1}\left(\gamma_{2} / \beta\right)\right.\right. \\
& \left.\left.-\left(\gamma_{2} / \beta\right)\right]\right\} /\left[(\pi / 2) R \gamma_{2}\right]^{1 / 2} \\
& \times \exp \left(-\gamma_{2} x_{1}\right)
\end{aligned}
$$

When both $J_{\beta R}$ and $H_{\beta R}{ }^{(1)}$ are replaced on the right-hand side of Eq. (B3) by the asymptotic expressions (B9) and (B12), respectively, the cancellation of all $R$-dependent factors is obvious, and we get

$$
\begin{aligned}
Q= & 1 / 2 \pi \int_{-\infty}^{+\infty} \mathrm{d} l_{y} \exp \left[i l_{y}\left(y-y^{\prime}\right)\right] \\
& \times \exp \left[\gamma_{2}\left(x_{1}{ }^{\prime}-x_{1}\right)\right] / 2 \gamma_{2} \\
= & 1 / 2 \pi \int_{-\infty}^{+\infty} \mathrm{d} l_{y} \exp \left[i l_{y}\left(y-y^{\prime}\right)\right] \\
& \times 1 / 2 \pi \int_{-\infty}^{+\infty} \mathrm{d} l_{x} \frac{\exp \left[i l_{x}\left(x_{1}-x_{1}{ }^{\prime}\right)\right]}{\left(l_{x}{ }^{2}+l_{y}{ }^{2}+\gamma^{2}\right)} .
\end{aligned}
$$

The last expression in Eq. (B13) follows from the fact that $x_{1}$ $-x_{1}^{\prime}>0$ and a residue integration. Finally, the combination of Eqs. (B13) and (4) leads immediately to the establishment of the validity of expression (B4). This proves that expression (B2) becomes Eq. (3) under the assumed condition, which was the announced check of consistency. Let us replace assumption (4) above in this appendix with

(4') $\bar{x}$ lies inside the waveguide, with $\theta$ close to $\pi / 2,|\bar{x}|=$ $\left(x^{2}+z^{2}\right)^{1 / 2} \simeq R+x_{1},\left|x_{1}\right|<R$ and $z \simeq R \varphi$ also hold.

Now, one is also led to expression (B2). If $x_{1}>x_{1}{ }^{\prime}, Q$ is still given by Eq. (B3), which, as before, can be shown to become expression (B4). If $x_{1}^{\prime}>x_{1}$, then $Q$ is given by the right-hand side of Eq. (B3), provided that the arguments of $H_{\beta} R^{(1)}$ and $J_{\beta R}$ be interchanged, and can also be approximated by expression (B4) [through a calculation similar to the one leading from Eq. (B3) to Eq. (B13), with $x_{1}$ and $x_{1}{ }^{\prime}$ interchanged]. Thus we conclude that, under the approximations (1)-(3) and assumption (4'), Eq. (5) also becomes Eq. (3), which constitutes another proof of consistency.

\section{ACKNOWLEDGMENTS}

M. L. Calvo acknowledges the partial financial support received from the Spain-USA Joint Committee for Scientific and Technical Cooperation and is grateful to Jay M. Enoch for his kind hospitality at the School of Optometry, University of California at Berkeley. R. F. Alvarez-Estrada acknowledges the partial financial support received from the Council for International Exchange of Scholars through a Fulbright-MEC Fellowship and is grateful to B. Zumino for his kind hospitality at the Theoretical Physics Group, Lawrence Berkeley Laboratory.

* Permanent address, Departamento de Optica, Facultad de Ciencias Físicas, Universidad Complutense, $28040 \mathrm{Ma}-$ drid, Spain.

† Permanent address, Departamento de Física Teorica, Facultad de Ciencias Físicas, Universidad Complutense, 28040 Madrid, Spain.

\section{REFERENCES}

1. E. A. J. Marcatili, "Bends in optical dielectric guides," Bell Syst. Tech. J. 48, 2103-2132 (1969); E. A. J. Marcatili and S. E. Miller, "Improved relations describing directional control in electromagnetic wave guidance," Bell Syst. Tech. J. 48, 2161-2187 (1969).

2. S. J. Maurer and L. B. Felsen, "Ray methods for trapped and slightly leaky modes in multilayered or multiwave regions," IEEE Trans. Microwave Theory Tech. MTT-18, 584-595 (1970).

3. L. Lewin, "Radiation from curved dielectric slabs and fibers," IEEE Trans. Microwave Theory Tech. MTT-22, 718-727 (1974).

4. J. A. Arnaud, "Transverse coupling in fiber optics III: bending losses," Bell Syst. Tech. J. 53, 1379-1394 (1974).

5. E. F. Kuester and D. C. Chang, "Surface wave radiation loss from curved dielectric slabs and fibers," IEEE J. Quantum Electron. QE-11, 903-907 (1975).

6. D. Marcuse, "Bent optical waveguides with lossy jacket," Bell Syst. Tech. J. 53, 1079-1101 (1974)

7. J. Sakai and T. Kimura, "Analytical bending loss formula of optical fibers with field deformation," Radio Sci. 17, 21-29 (1982).

8. D. Marcuse, "Curvature loss formula for optical fibers," J. Opt. Soc. Am. 66, 216-220 (1976)

9. D. Marcuse, "Field deformation and loss caused by curvature of optical fibers," J. Opt. Soc. Am. 66, 311-320 (1976).

10 D. Marcuse, "Influence of curvature on the losses of doubly clad fibers," Appl. Opt. 21, 4208-4213 (1982).

11. D. Marcuse, Theory of Dielectric Optical Waveguides (Academic, New York, 1974), Secs. 2.5 and 4.6.

12. D. Gloge, "Propagation effects in optical fibers," IEEE Trans. Microwave Theory Tech. MTT-23, 106-120 (1975); "Bending losses in multimode fibers with graded and ungraded core index," Appl. Opt. 11, 2506-2513 (1972).

13. Y. Takuma, M. Miyagi, and S. Kawakami, "Bent asymmetric dielectric slab waveguides," Appl. Opt. 20, 2291-2298 (1981); M. Miyagi, "Bending losses in hollow and dielectric tube leaky waveguides," Appl. Opt. 20, 1221-1229 (1981).

14. A. W. Snyder, J. A. White, and D. J. Mitchell, "Radiation from bent optical waveguides," Electron. Lett. 11, 332-333 (1975); J. A. White, "Radiation from bends in optical waveguides: the volume current method," Microwave Opt. Acoust. 3, 186-188 (1979).

15. J. I. Sakai, "Microbending loss evaluation in arbitrary-index single-mode optical fibers," IEEE J. Quantum Electron. QE-16, 36-49 (1980).

16. M. A. Miller and V. I. Talanov, "Electromagnetic surface waves guided by a boundary with small curvature," Z. Tekh. Fiz. 26, 2755 (1956). 
17. V. V. Shevchenko, "Radiation losses in bent waveguides for surface waves," Radiophys. Quantum Electron. 14, 607-614 (1973) (Russian original 1971).

18. W. A. Gambling, D. N. Payne, and H. Matsumura, "Radiation from curved single-mode fibers," Electron. Lett. 12, 567-569 (1976); W. A. Gambling, H. Matsumura, and C. M. Ragdale, "Field deformation in a curved single-mode fiber," Electron. Lett. 14, 130-132 (1978); W. A. Gambling, H. Matsumura, and C. M. Ragdale, "Curvature and microbending losses in singlemode optical fibers," Opt. Quantum Electron. 11, 43-59 (1979).

19. D. Marcuse, Light Transmission Optics (Van Nostrand, New York, 1972), Sec. 9.6.
20. A. W. Snyder and J. D. Love, Optical Waveguide Theory (Chapman and Hall, London, 1983), Chap. 23.

21. M. L. Calvo and R. F. Alvarez-Estrada, "Neutron fibres II: some improving alternatives and analysis of bending losses," J. Phys. D 19, 957-973 (1986).

22. P. M. Morse and H. Feschbach, Methods of Theoretical Physics (McGraw-Hill, New York, 1953), Vol. 1, Chap. 7, Sec. 2.

23. M. Abramowitz and I. Stegun, eds., Handbook of Mathematical Functions (Dover, New York, 1965).

24. A. Erdelyi, Asymptotic Expansions (Dover, New York, 1956), p. 36. 